\title{
An asteroseismic test of diffusion theory in white dwarfs
}

\author{
T. S. Metcalfe ${ }^{1}$, R. E. Nather ${ }^{2}$, T. K. Watson ${ }^{3}$, S.-L. Kim ${ }^{4}$, B.-G. Park $^{4}$, and G. Handler ${ }^{5}$ \\ ${ }^{1}$ High Altitude Observatory, National Center for Atmospheric Research, PO Box 3000, Boulder, CO 80307-3000, USA \\ e-mail: travis@hao.ucar.edu \\ 2 Department of Astronomy, University of Texas, Austin, TX 78712, USA \\ 3 Southwestern University, 1001 E. University Avenue, Georgetown, TX 78626, USA \\ 4 Korea Astronomy Observatory, Daejeon 305-348, Korea \\ 5 Institut für Astronomie, Universität Wien, Türkenschanzstraße 17, 1180 Wien, Austria \\ Received 23 December 2004 / Accepted 6 February 2005
}

\begin{abstract}
The helium-atmosphere (DB) white dwarfs are commonly thought to be the descendants of the hotter PG 1159 stars, which initially have uniform $\mathrm{He} / \mathrm{C} / \mathrm{O}$ atmospheres. In this evolutionary scenario, diffusion builds a pure He surface layer which gradually thickens as the star cools. In the temperature range of the pulsating DB white dwarfs $\left(T_{\text {eff }} \sim 25000 \mathrm{~K}\right)$ this transformation is still taking place, allowing asteroseismic tests of the theory. We have obtained dual-site observations of the pulsating DB star CBS 114, to complement existing observations of the slightly cooler star GD 358 . We recover the 7 independent pulsation modes that were previously known, and we discover 4 new ones to provide additional constraints on the models. We perform objective global fitting of our updated double-layered envelope models to both sets of observations, leading to determinations of the envelope masses and pure He surface layers that qualitatively agree with the expectations of diffusion theory. These results provide new asteroseismic evidence supporting one of the central assumptions of spectral evolution theory, linking the DB white dwarfs to PG 1159 stars.
\end{abstract}

Key words. stars: evolution - stars: individual: CBS 114 - stars: individual: GD 358 - stars: interiors - stars: oscillations white dwarfs

\section{Introduction}

The origin and evolution of the helium-atmosphere (DB) white dwarf stars has long been a subject of debate. They comprise roughly $20 \%$ of the population of field white dwarfs, with most of the remaining $80 \%$ consisting of their hydrogenatmosphere (DA) cousins. The majority of white dwarfs in both of these spectral classes are thought to arise from the evolution of isolated main-sequence stars with masses that are insufficient to ignite carbon fusion, which ultimately leave their hot carbon/oxygen cores to descend the white dwarf cooling track. The bifurcation into two spectral classes is thought to occur during post-asymptotic-giant-branch (post-AGB) evolution when, in some cases, a very late thermal pulse burns off the residual hydrogen in the envelope, producing a nearly pure helium atmosphere (Iben et al. 1983). Such objects are then supposed to return to the white dwarf cooling track as PG 1159 stars, which are widely believed to be the precursors of most DB white dwarfs.

The primary difficulty with this scenario is the paucity of DB white dwarfs with temperatures between 45000 and $30000 \mathrm{~K}-\mathrm{a}$ phenomenon known as the "DB gap" (Liebert et al. 1986), which persists even in the latest results from the Sloan Digital Sky Survey (Kleinman et al. 2004). If the PG 1159 stars and DB white dwarfs are linked, then why don't we see the long-lived intermediate phase? The spectral evolution theory advanced by Fontaine \& Wesemael (1987, 1997) postulates that traces of hydrogen $\left(M_{\mathrm{H}} \sim 10^{-15} M_{*}\right)$ in the envelopes of hot PG 1159 stars float to the surface and disguise them as DA stars within this temperature interval. A growing helium convection zone eventually dilutes the hydrogen in the photosphere until it is no longer detectable at $30000 \mathrm{~K}$, and the star continues its evolution as a DB. A relative overabundance of DA stars inside the DB gap supports this hypothesis (see Kleinman et al. 2004, their Fig. 11). But lower than predicted surface hydrogen abundances, measured for several DB stars just below the gap, remain a problem for spectral evolution theory (Provencal et al. 2000).

If we assume that there is an evolutionary connection between the PG 1159 stars and the cooler DB white dwarfs, we can look to several independent groups who have used time-dependent diffusion calculations to follow the changes in the interior structure of these objects as they cool (Dehner \& Kawaler 1995; Fontaine \& Brassard 2002; Althaus \& Córsico 2004). The hot PG 1159 stars, having recently emerged from the born-again phase, contain envelopes with a nearly uniform mixture of helium $(\mathrm{He})$, carbon $(\mathrm{C})$, and oxygen $(\mathrm{O})$ out to the photosphere (Dreizler \& Heber 1998; Herwig et al. 1999). As they cool, the He diffuses upward and gradually accumulates to form a chemically pure surface layer. This leads to a doublelayered structure, with the pure He surface layer overlying the 
remainder of the uniform $\mathrm{He} / \mathrm{C} / \mathrm{O}$ envelope, all above the degenerate $\mathrm{C} / \mathrm{O}$ core.

A key prediction of the diffusion models is that, for a given stellar mass, the pure $\mathrm{He}$ surface layer will steadily grow thicker as the DB star cools. The only available observational tests of this prediction come from asteroseismology - the study of the internal structure of stars through the interpretation of their pulsation periods. Fortunately, non-radial oscillations are naturally excited in otherwise normal DB white dwarf stars as they cool through a narrow range of temperatures near $25000 \mathrm{~K}$. However, there are only 9 such pulsating DB stars (DBVs) presently known, with effective temperatures between 22400 and $28400 \mathrm{~K}$ (Beauchamp et al. 1999).

The most thoroughly studied DBV star is GD 358, which has been the target of three coordinated observing campaigns of the Whole Earth Telescope (WET; Nather et al. 1990; Winget et al. 1994; Vuille et al. 2000; Kepler et al. 2003). These observations revealed dozens of independent pulsation periods, including a sequence of 11 dipole $(\ell=1, m=0$; Kotak et al. 2003) modes of consecutive radial overtone ( $k$; Bradley $\&$ Winget 1994), each providing a complementary probe of the stellar interior. These data formed the basis for the only asteroseismic tests of the diffusion models to date (Dehner \& Kawaler 1995; Fontaine \& Brassard 2002). In the most extensive of these tests, Fontaine \& Brassard (2002) produced a targeted grid of double-layered evolutionary models to try to fit the periods of GD 358 in detail. They found evidence of two composition transition zones near outer mass fractions of $10^{-5.8}$ and $10^{-3.0} M_{*}$, which they interpreted as the base of the pure $\mathrm{He}$ surface layer and the still-uniform $\mathrm{He} / \mathrm{C} / \mathrm{O}$ envelope, respectively. However, Montgomery et al. (2003) showed that there is a potential ambiguity in the inferred locations of these internal structures, due to an inherent core-envelope symmetry for models of DBV stars. As a consequence, real structure at specific locations in the core could be mis-interpreted as structure at certain symmetric points in the envelope, and vice versa.

The best way of circumventing this potential ambiguity is to fit additional stars using the same models, to determine whether the independent observations are consistent with the same underlying physical processes. Such model-fitting attempts for DBV stars other than GD 358 have been hampered by the lack of suitable observational data. Handler et al. (2002, hereafter HMW) found a total of 7 independent pulsation periods in the slightly hotter DBV star CBS 114, and Metcalfe et al. (2003) attempted to fit these observations using double-layered envelope models that were also applied to GD 358. They found qualitative agreement with the predictions of diffusion theory (thinner surface He layers for hotter white dwarfs of comparable mass) when using models that also included an adjustable $\mathrm{C} / \mathrm{O}$ profile in the core. However, the large number of free parameters in their model, relative to the number of observed periods in CBS 114, made these results somewhat uncertain.

\section{Observations and data reduction}

Motivated by the need for additional pulsation periods to constrain the model-fitting of CBS 114, we organized a dual-site campaign using $2 \mathrm{~m}$ class telescopes in February 2004. Our
Table 1. Journal of observations.

\begin{tabular}{llccc}
\hline \hline Run & Telescope & Date & $\begin{array}{c}\text { Start } \\
(\mathrm{UT})\end{array}$ & $\begin{array}{c}\text { Length } \\
(\mathrm{s})\end{array}$ \\
\hline 040219 & BOAO 1.8 m & 2004 Feb. 19 & $11: 15: 50$ & 33540 \\
040220 & BOAO 1.8 m & 2004 Feb. 20 & $10: 58: 19$ & 13080 \\
A0844 & McD 2.1 m & 2004 Feb. 21 & $04: 17: 05$ & 11050 \\
040223 & BOAO 1.8 m & 2004 Feb. 23 & $10: 42: 57$ & 29520 \\
040224 & BOAO 1.8 m & 2004 Feb. 24 & $15: 58: 30$ & 14520 \\
040225 & BOAO 1.8 m & 2004 Feb. 25 & $12: 49: 33$ & 24840 \\
A0847 & McD 2.1 m & 2004 Feb. 26 & $01: 53: 07$ & 36120 \\
A0848 & McD 2.1 m & 2004 Feb. 27 & $01: 51: 17$ & 38550 \\
\hline
\end{tabular}

primary goals were to confirm the periods of the 7 pulsation modes found by HMW, and to discover additional modes below the $\sim 5$ milli-mag noise limit of the previous observations (obtained over 3 weeks from the $0.75 \mathrm{~m}$ telescope at SAAO). This campaign was awarded 7 nights (2004 Feb. 19-25) on the $1.8 \mathrm{~m}$ telescope at Bohyunsan Optical Astronomy Observatory (BOAO) in Korea, and 7 nights (2004 Feb. 21-27) on the $2.1 \mathrm{~m}$ telescope at McDonald Observatory (McD) in Texas.

At BOAO we used a thinned SITe $2 \mathrm{~K}$ CCD camera with no filter. To minimize the readout time, we binned the pixels $2 \times 2$ for an effective resolution of $0.68 \mathrm{arcsec}_{\mathrm{pixel}}{ }^{-1}$, and we used only the central portion of the detector for a field of view $\sim 4$ arcmin on a side. On the first night we used an exposure time of $8 \mathrm{~s}$ for a cycle time of $20 \mathrm{~s}$, but on subsequent nights we increased the exposure time to $20.5 \mathrm{~s}$ for a cycle time of $30 \mathrm{~s}$. This system has a gain of $1.8 \mathrm{e}^{-} \mathrm{ADU}^{-1}$ and a readout noise of $7 \mathrm{e}^{-}$rms.

The observations at $\mathrm{McD}$ were obtained using the Argos time-series CCD photometer (Nather \& Mukadam 2004) with no filter. This instrument uses a back-illuminated $512 \times 512$ frame-transfer CCD mounted at the $\mathrm{f} / 3.9$ prime focus, for a field of view 2.8 arcmin on a side and a resolution of 0.33 arcsec pixel $^{-1}$. We used an exposure time of $10 \mathrm{~s}$, which was also the cycle time - the transfer of each image to the masked on-chip buffer requires only $310 \mu \mathrm{s}$, and the $280 \mathrm{~ms}$ readout takes place during the next exposure. This system has a gain of $2 \mathrm{e}^{-} \mathrm{ADU}^{-1}$ and a readout noise of $8 \mathrm{e}^{-} \mathrm{rms}$. The observations collected for this dual-site campaign are summarized in Table 1.

The raw CCD frames were analyzed on-the-fly during the observing campaign to monitor the data quality, but final reductions were done uniformly using the IRAF high-speed photometry (HSP) package developed by Antonio Kanaan (personal communication). After correcting each image for bias level and flat field effects, we extracted aperture photometry for CBS 114 and three nearby comparison stars. The aperture was typically set to have a radius between 2 and 4 arcsec with a surrounding annulus to determine the local sky level, but the apertures were adjusted for each data set to maximize the $\mathrm{S} / \mathrm{N}$ of the resulting light curves. The time-series measurements were then imported into XQED (Riddle 2003), the photometry analysis software for the WET. For each light curve, we subtracted the local sky contribution and performed a point-by-point division of the target star by the average of the three comparison stars. We then corrected for any low-frequency variations (e.g., caused by 


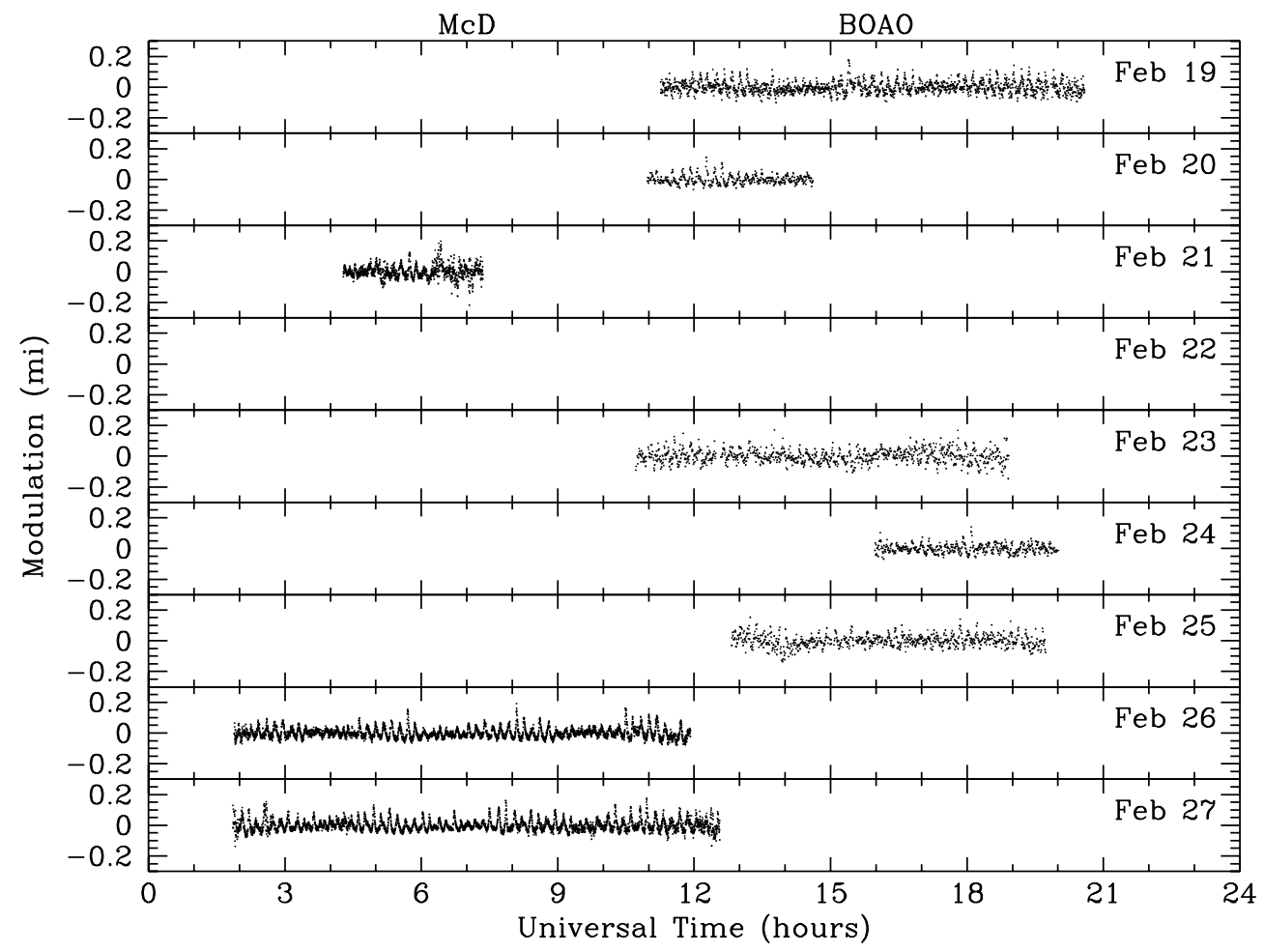

Fig. 1. Reduced light curves for CBS 114 during our dual-site campaign in 2004 , showing fractional intensity variations of \pm 15 percent with a dominant period of $\sim 650 \mathrm{~s}$.

differential color extinction) by fitting and removing a secondorder polynomial. Finally, we subtracted the mean light level and applied barycentric timing corrections to the exposure midtimes. The resulting light curves for CBS 114 are shown in Fig. 1.

\section{Frequency analysis}

The Fourier Transforms (FTs) of these light curves, for each observatory and for the combined data set, are shown in Fig. 2. The corresponding window functions - the FT of a single sinusoid sampled in the same way as the data - are shown to the same scale in each panel. The distributed sampling of the BOAO data yields better effective frequency resolution, while the slightly larger aperture and higher cadence of the McD data leads to a higher $\mathrm{S} / \mathrm{N}$. The full data set combines all of these strengths, and reduces the amplitude of the $1 d$ aliases (separated from the main frequency by $\pm 11.6 \mu \mathrm{Hz}$ ).

To determine the significance level of peaks in each of these FTs, we followed the analysis of Kepler (1993). Since power is the square of the amplitude, the average power out to the Nyquist frequency is a measure of the mean-square deviation from zero amplitude, which is dominated by the noise since the signal is confined to a relatively narrow range of frequencies. The square-root of this value is the root-mean-square amplitude of the noise, which is analogous to the standard deviation $\left(\sigma_{\text {noise }}\right)$. By adopting a significance cut at $3 \sigma_{\text {noise }}$, we ensure that only $\sim 0.3 \%$ of the peaks with this amplitude will not be real signals (assuming the noise is Gaussian). Peaks with amplitudes much above the significance cut, and those detected in multiple independent data sets, have an even lower chance of being due to noise. The $3 \sigma_{\text {noise }}$ significance level is indicated by the dotted line in each panel of Fig. 2, which is at 3.52, 2.33, and $2.26 \mathrm{mma}$ for the $\mathrm{BOAO}, \mathrm{McD}$, and combined data sets respectively. For the range of frequencies shown, we expect only $\sim 4$ noise peaks to appear above these cuts.

We performed a frequency analysis of the combined data set using the program PERIOD98 (Sperl 1998). We started by iteratively fitting and subtracting the frequencies with the largest amplitudes, refining our estimates of the period, amplitude, and phase for each signal using simultaneous multiple sine-wave fitting. This allowed us to recover the 7 independent pulsation frequencies found by HMW, with a few important differences. We resolved both of the two largest amplitude modes near 1520 and $1610 \mu \mathrm{Hz}$ into triplets, though the splitting is close to the $1 d$ aliases (perhaps explaining why the earlier single-site data failed to detect them, despite a longer time baseline). We also detected an additional component in the $1520 \mu \mathrm{Hz}$ multiplet at $1514.1 \mu \mathrm{Hz}$, which could be caused by amplitude and/or frequency modulation during the observations or might be caused by higher-order combination frequencies, comparable to the " $k=\hat{15}$ " mode seen in GD 358 in 1994 (Vuille et al. 2000). Our frequencies for the modes near 1830 and $1960 \mu \mathrm{Hz}$ are both systematically lower than in HMW, but by less than would be expected from $1 d$ alias problems. This might be the result of an underlying multiplet structure with intrinsic amplitude modulation among the various components (e.g., see Robinson et al. 1976, their Fig. 2). We also found that our mode near $2300 \mu \mathrm{Hz}$ was the $+1 d$ alias of the frequency found by HMW - a possibility that they could not rule out. Finally, we resolved the mode near $2510 \mu \mathrm{Hz}$, as well as 


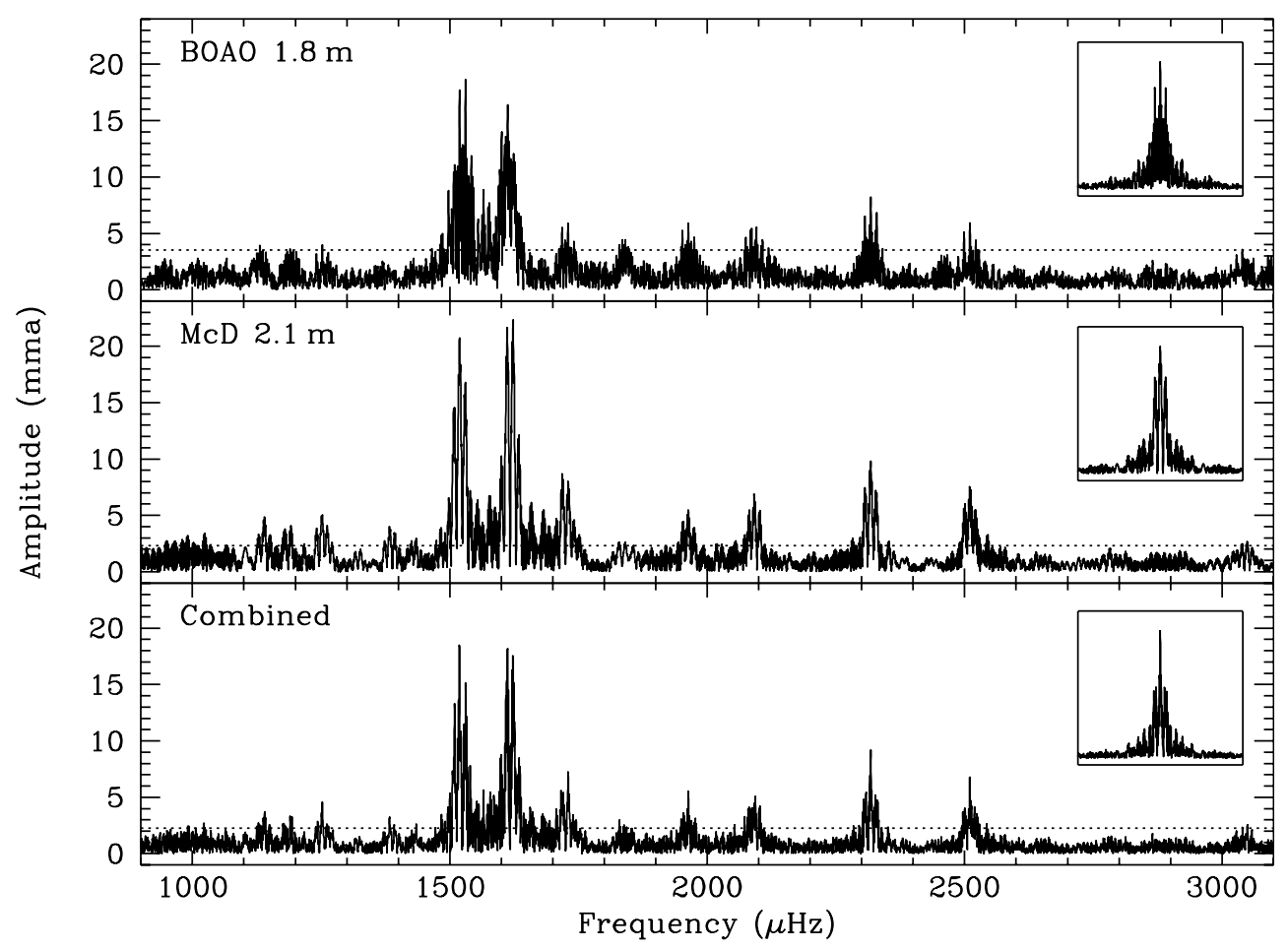

Fig. 2. Fourier Transforms (FTs) of the light curves shown in Fig. 1 over the range of independent frequencies exhibited by CBS 114 . The three panels show the FTs for the data from BOAO (top), $\mathrm{McD}$ (middle), and for the combined data set (bottom), along with the corresponding window functions (inset) and significance cuts (dotted line).

a newly discovered mode near $2090 \mu \mathrm{Hz}$, into doublets with a splitting comparable to the frequency shifts seen in the 1830 and $1960 \mu \mathrm{Hz}$ modes.

In addition to recovering the 7 pulsation modes found by HMW, we also discovered 4 new ones. The largest is the doublet near $2090 \mu \mathrm{Hz}$, which nicely fills the gap in the sequence of consecutive radial overtones identified by HMW. A marginal peak near this frequency also appeared in the discovery data of Winget \& Claver (1988, 1989), but it was below the detection threshold of HMW. The three other new modes appear at frequencies near 1190, 1250, and $1380 \mu \mathrm{Hz}$. The period spacing of these peaks suggests that they are additional independent pulsation modes with higher radial overtones than those previously seen. Two additional frequencies had amplitudes near our significance threshold. These could tentatively be identified as an even higher radial overtone near $1140 \mu \mathrm{Hz}$, and a possible rotationally split component of the mode near $1720 \mu \mathrm{Hz}$. These marginal detections prompted us to compute weighted FTs, following the suggestion of Handler (2003). The results were nearly identical to the unweighted case, and the marginal nature of these two frequencies was unchanged.

Aside from the independent pulsation modes observed in CBS 114, we also detected 11 significant linear combination frequencies. Fundamentally, these frequencies arise in our analysis because the Fourier Transform decomposes the signal into sinusoidal components. A casual inspection of the light curves shown in Fig. 1 reveals that they are not perfectly sinusoidal. Instead, they tend to have sharp peaks and shallow troughs, which is commonly interpreted to be associated with a nonlinear response of the surface convection zone to the pulsations (e.g., see Brickhill 1992; Wu 2001; Ising \& Koester 2001; Montgomery 2005). The earlier observations by HMW revealed two such frequencies, both of which are among those we detected. The combination frequencies identified in our data involve the six modes or multiplets with the largest amplitudes.

Our final simultaneous fit of 28 sinusoids to the data using PERIOD98 contains a total of 17 independent modes and 11 linear combination frequencies, which are all listed in Table 2. In Fig. 3 we show the FT of our combined data set before and after the removal of these 28 frequencies (indicated by solid and open triangles for the independent and combination frequencies, respectively). The marginal signals near 1140 , 1514 , and $1720 \mu \mathrm{Hz}$ are apparent in the residuals.

\section{Model fitting}

To investigate whether these new observations can fit comfortably with the predictions of diffusion theory, we repeated the global model-fitting procedure described by Metcalfe et al. (2003). We used an updated version of the code that incorporates the OPAL radiative opacities (Iglesias \& Rogers 1996) rather than the older LAO data (Huebner et al. 1977), which are known to produce systematic errors in the derived temperatures (Fontaine \& Brassard 1994). The fitting procedure uses a parallel genetic algorithm (Metcalfe \& Charbonneau 2003) to minimize the root-mean-square residuals between the observed and calculated periods $\left(\sigma_{\mathrm{P}}\right)$ for models with effective temperatures $\left(T_{\text {eff }}\right)$ between 20000 and $30000 \mathrm{~K}$, and stellar masses $\left(M_{*}\right)$ between 0.45 and $0.95 M_{\odot}$. It allows the base of the uniform $\mathrm{He} / \mathrm{C}$ envelope to be located at an outer mass 


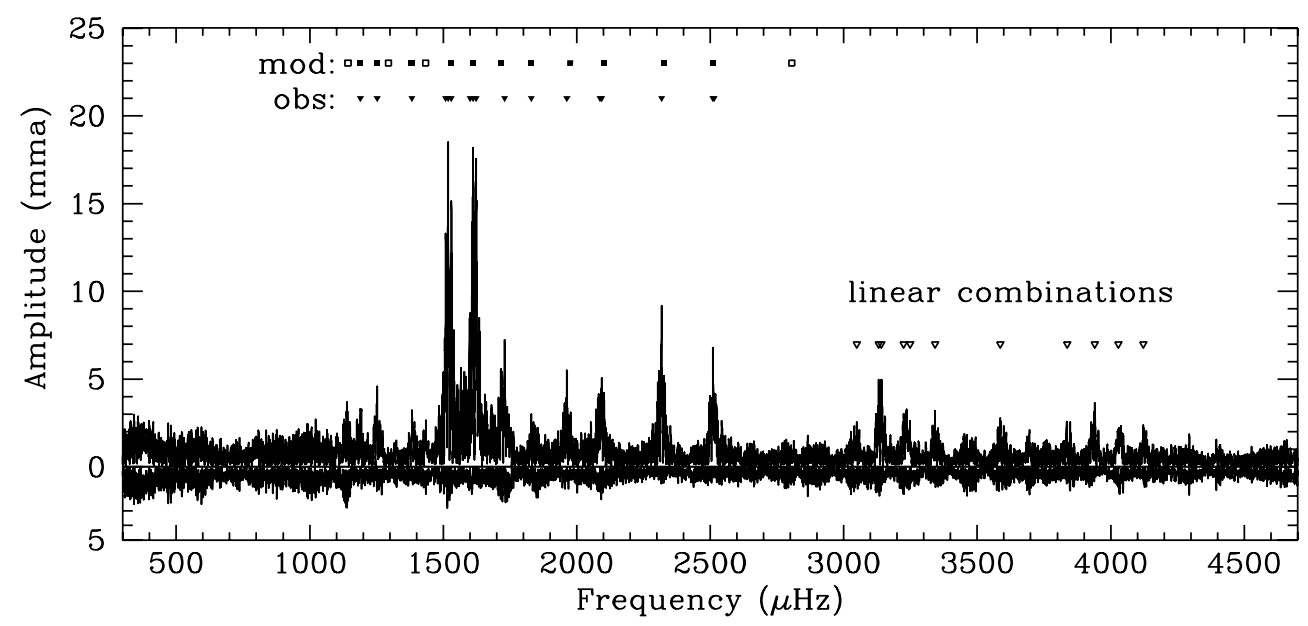

Fig. 3. The Fourier Transform of our combined data set for CBS 114 before and after removing the 28 sinusoids listed in Table 2. The independent and linear combination frequencies are indicated by the solid and open triangles respectively. The frequencies of our optimal model matched to the observed modes are shown as solid squares, while open squares denote model frequencies with no detected counterpart.

Table 2. The multifrequency solution for CBS 114 from PERIOD98. Also shown is the mode identification from our optimal model.

\begin{tabular}{|c|c|c|c|c|c|c|}
\hline \multirow[t]{2}{*}{ Sinusoid } & \multirow{2}{*}{$\begin{array}{c}\text { Frequency } \\
(\mu \mathrm{Hz})\end{array}$} & \multirow{2}{*}{$\begin{array}{c}\text { Period } \\
\text { (s) }\end{array}$} & \multirow{2}{*}{$\begin{array}{l}\text { Amp } \\
\text { (mmag) }\end{array}$} & \multicolumn{3}{|c|}{ "Identification } \\
\hline & & & & $k$ & $\ell$ & $m$ \\
\hline$f_{1} \ldots \ldots$ & 1189.9 & 840.38 & 3.1 & 20 & 1 & 0 \\
\hline$f_{2} \ldots \ldots$ & 1252.4 & 798.46 & 4.3 & 19 & 1 & 0 \\
\hline$f_{3} \ldots \ldots$ & 1383.0 & 723.06 & 3.0 & 17 & 1 & 0 \\
\hline$f_{4} \ldots \ldots$ & 1509.0 & 662.67 & 7.5 & 15 & 1 & -1 \\
\hline$f_{5} \ldots \ldots$ & 1518.8 & 658.44 & 11.8 & 15 & 1 & 0 \\
\hline$f_{6} \ldots \ldots$ & 1530.7 & 653.30 & 9.0 & 15 & 1 & +1 \\
\hline$f_{7} \ldots \ldots$ & 1601.2 & 624.55 & 4.4 & 14 & 1 & -1 \\
\hline$f_{8} \ldots \ldots$ & 1612.1 & 620.31 & 15.4 & 14 & 1 & 0 \\
\hline$f_{9} \ldots \ldots$ & 1622.7 & 616.27 & 13.3 & 14 & 1 & +1 \\
\hline$f_{10} \ldots \ldots$ & 1729.8 & 578.09 & 7.0 & 13 & 1 & 0 \\
\hline$f_{11} \ldots \ldots$ & 1829.6 & 546.56 & 3.0 & 12 & 1 & 0 \\
\hline$f_{12} \ldots \ldots$ & 1963.2 & 509.37 & 5.5 & 11 & 1 & 0 \\
\hline$f_{13} \ldots \ldots$ & 2086.5 & 479.27 & 4.8 & 10 & 1 & $?$ \\
\hline$f_{14} \ldots \ldots$ & 2093.6 & 477.65 & 4.9 & 10 & 1 & $?$ \\
\hline$f_{15} \ldots \ldots$ & 2317.7 & 431.45 & 8.9 & 9 & 1 & 0 \\
\hline$f_{16} \ldots \ldots$ & 2510.0 & 398.41 & 6.8 & 8 & 1 & $?$ \\
\hline$f_{17} \ldots \ldots$ & 2515.2 & 397.59 & 3.2 & 8 & 1 & $?$ \\
\hline$f_{5}+f_{6} \ldots$ & 3049.4 & 327.93 & 2.2 & $\ldots$ & $\ldots$ & $\ldots$ \\
\hline$f_{5}+f_{8} \ldots$ & 3130.9 & 319.40 & 3.7 & $\ldots$ & $\cdots$ & $\ldots$ \\
\hline$f_{5}+f_{9} \ldots$ & 3141.4 & 318.33 & 3.5 & $\ldots$ & $\ldots$ & $\ldots$ \\
\hline$f_{8}+f_{8} \ldots$ & 3224.2 & 310.15 & 2.4 & $\ldots$ & $\ldots$ & $\ldots$ \\
\hline$f_{5}+f_{10} \ldots$ & 3248.6 & 307.83 & 2.2 & $\ldots$ & $\ldots$ & $\ldots$ \\
\hline$f_{8}+f_{10} \ldots$ & 3341.9 & 299.23 & 3.2 & $\ldots$ & $\ldots$ & $\ldots$ \\
\hline$f_{9}+f_{12} \ldots$ & 3585.9 & 278.87 & 2.9 & $\ldots$ & $\ldots$ & $\ldots$ \\
\hline$f_{5}+f_{15} \ldots$ & 3836.5 & 260.65 & 2.4 & $\ldots$ & $\ldots$ & $\ldots$ \\
\hline$f_{9}+f_{15}$ & 3940.4 & 253.78 & 3.3 & $\ldots$ & $\ldots$ & $\ldots$ \\
\hline$f_{5}+f_{16} \ldots$ & 4028.8 & 248.22 & 2.0 & $\ldots$ & $\ldots$ & $\ldots$ \\
\hline$f_{8}+f_{16} \ldots$ & 4122.1 & 242.59 & 2.1 & $\cdots$ & $\ldots$ & $\ldots$ \\
\hline
\end{tabular}

fraction $\log \left(M_{\text {env }} / M_{*}\right)$ between -2.0 and -4.0 . The base of the pure He surface layer can assume values of $\log \left(M_{\mathrm{He}} / M_{*}\right)$ between -5.0 and -7.0 . To ensure a smooth transition between the self-consistent cores and the static envelopes in our
Table 3. Optimal model parameters for CBS 114 and GD 358.

\begin{tabular}{lrrl}
\hline \hline Parameter & CBS 114 & GD 358 & Error \\
\hline$T_{\text {eff }}(\mathrm{K}) \ldots \ldots \ldots$ & 25800 & 23100 & \pm 100 \\
$M_{*}\left(M_{\odot}\right) \ldots \ldots$ & 0.630 & 0.630 & \pm 0.005 \\
$\log \left(M_{\text {env }} / M_{*}\right) \ldots$ & -2.42 & -2.92 & \pm 0.02 \\
$\log \left(M_{\mathrm{He}} / M_{*}\right) \ldots$ & -5.96 & -5.90 & \pm 0.02 \\
$\sigma_{\mathrm{P}}(\mathrm{s}) \ldots \ldots \ldots$ & 2.33 & 2.26 & $\ldots$ \\
\hline
\end{tabular}

models, we fixed the core composition to be pure C. We cannot presently include oxygen in the envelopes of our models.

We applied this fitting procedure to our new observations of CBS 114 and to the WET observations of GD 358 from 1990 (Winget et al. 1994) for comparison. For CBS 114 we assumed that each single mode had an azimuthal order $m=0$ (see Metcalfe 2003a), and we used the central frequency for each of the observed triplets. For the two doublets, we used the average of the two observed frequencies. The resulting sets of optimal model parameters for these two stars are shown in Table 3 with internal errors for each parameter set by the resolution of our search. The theoretical pulsation frequencies for CBS 114 are shown in Fig. 3 as solid squares where they match an observed frequency, and the corresponding mode identifications are listed in Table 2. For reference, the open squares in Fig. 3 show additional model frequencies with no clearly detected counterpart.

The spectroscopic temperature determinations of Beauchamp et al. (1999) suggest that CBS 114 could be as much as $1500 \mathrm{~K}$ hotter than GD 358. Our model-fitting results suggest a larger temperature difference, placing CBS 114 at $2700 \pm 200 \mathrm{~K}$ hotter than GD 358. On an absolute scale, the temperature and mass of our fit to CBS 114 are consistent with the spectroscopic values, assuming that any traces of $\mathrm{H}$ in the atmosphere are small. Our fit to GD 358 is consistent with the spectroscopic mass, but our temperature determination is slightly low. Our value is also low compared to a more recent and precise determination using UV spectroscopy from the 
Hubble Space Telescope (24 $100 \pm 400 \mathrm{~K}$; Castanheira et al. 2005).

Recent time-dependent diffusion calculations by Althaus \& Córsico (2004) suggest that for a given stellar mass and envelope thickness, the pure He surface layer should thicken by $\sim 0.15$ dex between the spectroscopic temperatures of CBS 114 and GD 358. We find a marginal thickening of only $0.06 \pm$ 0.04 dex. However, diffusion models with more massive envelopes lead to thicker surface He layers more quickly as the star cools, since there is a larger reservoir of He to draw from. Our fit to CBS 114 has a slightly larger total envelope mass, which would tend to diminish the expected difference between the thickness of the surface He layers for the two stars to $\sim 0.12$ dex. The limited grid of published diffusion models currently makes more quantitative comparisons impossible.

\section{Discussion}

Our new dual-site observations of the pulsating DB white dwarf CBS 114 have finally revealed enough independent modes to bring the model-fitting for this star to the same level of reliability as for GD 358. We used our updated double-layered envelope models to match the new observations of CBS 114, and the archival observations of GD 358 - fitting 11 pulsation modes in each star. The resulting pair of optimal models have pure $\mathrm{He}$ surface layers and total envelope masses that qualitatively agree with our expectations from diffusion theory. This provides new asteroseismic evidence supporting one of the central assumptions of spectral evolution theory, linking the DB white dwarfs to PG 1159 stars.

In addition to the new observational constraints for CBS 114, we improved our analysis by incorporating the OPAL radiative opacities into the models. Our results include higher temperatures and lower masses than the previous fits by Metcalfe et al. (2003), leading to better agreement with the spectroscopic estimates for both stars. The optimal locations for the base of the pure He surface layer and the uniform $\mathrm{He} / \mathrm{C}$ envelope are systematically deeper for GD 358 relative to the previous fit. For CBS 114 the fit is now consistent with the envelope mass expected from simulations of carbon dredge-up in DQ stars (see Pelletier et al. 1986; Fontaine \& Brassard 2002), and with the He layer mass expected from time-dependent diffusion calculations (Althaus \& Córsico 2004).

These results have now met the challenge posed by Metcalfe (2003b), to fit the observations of more than one DBV star and demonstrate that they are both consistent with the same underlying physical process. In that paper, the relevant process was the nuclear burning history during the red giant phase leading to a $\mathrm{C} / \mathrm{O}$ ratio in the core that could help to constrain the rate of the ${ }^{12} \mathrm{C}(\alpha, \gamma){ }^{16} \mathrm{O}$ reaction. In the present case, the relevant process is diffusion in the white dwarf envelopes leading to a gradually thickening pure He surface layer as the star cools. In effect, each of these studies has concentrated on an isolated structure in the stellar interior, ignoring the contribution of other possible features. Regardless of whether the asteroseismic signal originates from the core or the envelope, it is now clear that the relevant structure $(\mathrm{C} / \mathrm{O}$ profile or
He surface layer) is very nearly the same in the two stars. The better absolute quality of the fits for GD 358 and CBS 114 in Metcalfe (2003b) suggest that the $\mathrm{C} / \mathrm{O}$ profile is the more important structure from an asteroseismic standpoint. Indeed, this is consistent with the finding by Montgomery et al. (2003) that the pure He surface layer has a smaller effect on the pulsation modes than the uniform $\mathrm{He} / \mathrm{C}$ envelope, which is itself secondary in importance to the $\mathrm{C} / \mathrm{O}$ profile in the core.

Of course, there are sound physical reasons to expect complicated structures in both the core and the envelope of real white dwarfs. We have demonstrated that analyses of two completely independent sets of pulsation data with two physically distinct classes of models can both lead to reasonably selfconsistent interpretations of the corresponding internal structures. Our challenge is now to synthesize hybrid models that can fit the core and envelope structure simultaneously. It is possible that such models will exhaust the information content of the 11 pulsation modes we have observed in these two stars. If so, our only recourse is to detect additional modes in GD 358 and CBS 114 and to discover additional DBV stars with rich pulsation spectra. Both options will probably require multi-site campaigns using $4 \mathrm{~m}$ class telescopes since the existing observations are already the result of campaigns using 1-2 m telescopes, and the newly discovered DBV stars from the Sloan Digital Sky Survey are significantly fainter (Nitta et al. 2005). Even so, the future continues to look bright for white dwarf asteroseismology.

Acknowledgements. We would like to thank Steve Kawaler and Mike Montgomery for their comments and suggestions. This work was partially supported by the Smithsonian Institution through a CfA Postdoctoral Fellowship, and by the National Science Foundation through an Astronomy \& Astrophysics Postdoctoral Fellowship under award AST-0401441. Computational resources were provided by White Dwarf Research Corporation through grants from the Fund for Astrophysical Research and from the American Astronomical Society.

\section{References}

Althaus, L. G., \& Córsico, A. H. 2004, A\&A, 417, 1115

Beauchamp, A., Wesemael, F., Bergeron, P., et al. 1999, ApJ, 516, 887

Bradley, P. A., \& Winget, D. E. 1994, ApJ, 430, 850

Brickhill, A. J. 1992, MNRAS, 259, 519

Castanheira, B. G., Nitta, A., Kepler, S. O., Winget, D. E., \& Koester, D. 2005, A\&A, 432, 175

Dehner, B. T., \& Kawaler, S. D. 1995, ApJ, 445, L141

Dreizler, S., \& Heber, U. 1998, A\&A, 334, 618

Fontaine, G., \& Brassard, P. 1994, in The Equation of State in Astrophysics, IAU Coll., 147, 347

Fontaine, G., \& Brassard, P. 2002, ApJ, 581, L33

Fontaine, G., \& Wesemael, F. 1987, in Second Conference on Faint Blue Stars, IAU Coll., 95, 319

Fontaine, G., \& Wesemael, F. 1997, in White dwarfs, ASSL, 214, 173 Handler, G. 2003, Baltic Astron., 12, 253

Handler, G., Metcalfe, T. S., \& Wood, M. A. 2002, MNRAS, 335, 698 [HMW]

Herwig, F., Blöcker, T., Langer, N., \& Driebe, T. 1999, A\&A, 349, L5

Huebner, W. F., Merts, A. L., Magee, N. H., \& Argo, M. F. 1977, Los Alamos Scientific Laboratory report, LA-6760-M

Iben, I., Kaler, J. B., Truran, J. W., \& Renzini, A. 1983, ApJ, 264, 605

Iglesias, C. A., \& Rogers, F. J. 1996, ApJ, 464, 943 
Ising, J., \& Koester, D. 2001, A\&A, 374, 116

Kepler, S. O. 1993, Baltic Astron., 2, 515

Kepler, S. O., Nather, R. E., Winget, D. E., et al. 2003, A\&A, 401, 639

Kleinman, S. J., Harris, H. C., Eisenstein, D. J., et al. 2004, ApJ, 607, 426

Kotak, R., van Kerkwijk, M. H., Clemens, J. C., \& Koester, D. 2003, A\&A, 397, 1043

Liebert, J., Wesemael, F., Hansen, C. J., et al. 1986, ApJ, 309, 241

Metcalfe, T. S. 2003a, Baltic Astron., 12, 247

Metcalfe, T. S. 2003b, ApJ, 587, L43

Metcalfe, T. S., \& Charbonneau, P. 2003, J. Comput. Phys., 185, 176

Metcalfe, T. S., Montgomery, M. H., \& Kawaler, S. D. 2003, MNRAS, 344, L88

Montgomery, M. H. 2005, ApJ, submitted

Montgomery, M. H., Metcalfe, T. S., \& Winget, D. E. 2003, MNRAS, 344,657

Nather, R. E., \& Mukadam, A. S. 2004, ApJ, 605, 846
Nather, R. E., Winget, D. E., Clemens, J. C., Hansen, C. J., \& Hine, B. P. 1990, ApJ, 361, 309

Nitta, A., Kleinman, S. J., Krzesinski, J., et al. 2005, in Proc. 14th European Workshop on White Dwarfs, ASP Conf., 334

Pelletier, C., Fontaine, G., Wesemael, F., Michaud, G., \& Wegner, G. 1986, ApJ, 307, 242

Provencal, J. L., Shipman, H. L., Thejll, P., \& Vennes, S. 2000, ApJ, 542, 1041

Riddle, R. L. 2003, Baltic Astron., 12, 183

Robinson, E. L., Nather, R. E., \& McGraw, J. T. 1976, ApJ, 210, 211

Sperl, M. 1998, Commun. Asteroseismol., 111, 1

Vuille, F., O'Donoghue, D., Buckley, D. A. H., et al. 2000, MNRAS, 314, 689

Winget, D. E., \& Claver, C. F. 1988, IAU Circ., 4595, 3

Winget, D. E., \& Claver, C. F. 1989, Lect. Notes Phys. (Berlin: Springer Verlag), 328, 290

Winget, D. E., Nather, R. E., Clemens, J. C., et al. 1994, ApJ, 430, 839

Wu, Y. 2001, MNRAS, 323, 248 